\title{
A Polymorphism in the Cyclooxygenase 2 Gene in Type 1 Diabetic Patients With Nephropathy
}

\author{
J. A. HUBÁČEK ${ }^{1}$, T. PELIKÁNOVÁ ${ }^{1}$, V. LÁNSKÁ ${ }^{1}$, R. KOMERS ${ }^{1}$ \\ ${ }^{1}$ Diabetes Center, Institute for Clinical and Experimental Medicine, Prague, Czech Republic
}

Received April 5, 2010

Accepted June 24, 2010

On-line November 29, 2010

\section{Summary}

Diabetic nephropathy (DN), the most serious complication of Type 1 diabetes (DM1), has a strong genetic component. Cyclooxygenase-2 (COX-2), an inducible enzyme by a number of stimuli, has been implicated in pathophysiology of cardiovascular and renal disease, including $\mathrm{DN}$. The allele $-765 \mathrm{C}$, of the $-765 \mathrm{G}>\mathrm{C}$ polymorphism (rs20417) in the COX-2 promoter has lower promoter activity compared with the $-765 \mathrm{G}$ allele and protective effects in cardiovascular disease. This polymorphism was not investigated in patients with DM1 and nephropathy. The study was conducted in 779 Caucasian patients with DM1 and compared to a representative sample of healthy Czech population. The patients demonstrated lower frequencies of the $C C$ genotype $(P=0.005)$. From the DM1 cohort, 153 patients met the criteria for low risk of the development of DN (LRDN, duration of DM1>10 years, normoalbuminuria, normotension) and 139 patients had manifest DN. There were no differences in $-765 \mathrm{G}>\mathrm{C}$ polymorphisms between LRDN and DN patients. Moreover, the $C / G$ allele frequencies did not also differ between the groups. In conclusion, patients with DM1 display lower freqencies of the protective CC genotype as compared to healthy subjects. However, the study did not reveal associations of $-765 \mathrm{G}>\mathrm{C}$ polymorphism with the risk of $\mathrm{DN}$.

\section{Key words}

Cyclooxygenase-2 • Diabetic nephropathy - -765G >C polymorphism

\section{Corresponding author}

R. Komers, Division of Nephrology and Hypertension, Oregon Health \& Science University, 3314 SW US Veterans Hospital Hospital Rd., 97239 Portland, OR, USA. E-mail: komersr@ohsu.edu

\section{Introduction}

Diabetic nephropathy (DN) is the most serious complication of Type 1 diabetes (DM1). Studies suggest strong contribution of genetic factors in the pathophysiology of DN. Therefore, identification of genetic markers that could help to identify diabetic patients susceptible to $\mathrm{DN}$ has become one of major issues in this area of research.

Cyclooxygenase $(\mathrm{COX})$ is the rate-limiting enzyme in the biosynthesis of prostaglandins, which catalyses the first step in the biosynthesis of prostaglandins by converting arachidonic acid to prostaglandin-H2 (Smith 1989). Prostaglandin-H2 is then rapidly converted into a variety of eicosanoids with a plethora of physiological and pathophysiological functions (Smith 1989). COX exists in two isoforms, COX-1 and COX-2, which are encoded by two separate genes (reviewed by Linton et al. 2002). COX-1 is constitutively expressed in most tissues mediating 'housekeeping' functions of the cells (Linton et al. 2002). In contrast, COX-2 operates as an inducible enzyme with low or undetectable levels in most tissues, and its expression can be markedly increased by a number of inflammatory, mitogenic, and physical stimuli (Linton et al. 2002).

Cyclooxygenase- 2 has been implicated in cardiovascular and renal pathophysiology, including the processes leading to the development of DN (Komers et al. 2001, Cherney et al. 2008). There is a large number of described variants within the human COX-2 gene. Most of these polymorphisms are intronic with unlikely impact 
on COX-2 enzymatic activity. However, a limited number of polymorphisms have been found in the COX-2 promoter region, with potential impact on COX-2 expression and activity. In 2002, Papafili et al. (2002) identified a new variant in the COX-2 promoter $-765 \mathrm{G}>\mathrm{C}$ (rs 20417), and showed that the variant $-765 \mathrm{C}$ has significantly lower $(-30 \%)$ promoter activity compared with the $-765 \mathrm{G}$ allele in vitro. Importantly, in patients undergoing coronary artery bypass surgery, the magnitude of rise in systemic markers of inflammation was strongly dependent on $-765 \mathrm{G}>\mathrm{C}$ genotype. In accord with this original observation, more recent studies demonstrated protective effect of $-765 \mathrm{C}$ in $\mathrm{CV}$ disease in association with lower molecular markers of vascular COX-2 activity and inflammation (Cipollone et al. 2004, Furukado et al. 2009).

Considering the putative roles of COX-2-derived prostanoids in the development of vascular complications of diabetes, functional polymorphisms in COX-2 gene could at least in part explain genetic susceptibility to DN. To explore this hypothesis, we determined the $-765 \mathrm{G}>\mathrm{C}$ polymorphism in the promoter region of COX-2 gene in Type 1 diabetic patients with and without nephropathy.

This study is part of the ongoing project in adult patients with diabetes patients treated in the Institute for Clinical and Experimental Medicine aiming at association of genetic variants and new disease markers in a large cohort with well defined phenotype. Seven hundred and seventy nine Type 1 diabetic patients were recruited between 2006 and 2009. The Type 1 diabetic patients were required to be C-peptide negative $(<0.2 \mathrm{nmol} / \mathrm{l})$ and to have permanent insulin treatment initiated before the age of 40 years, within 1 year of the diabetes diagnosis. To determine distribution of COX-2 $-765 \mathrm{G}>\mathrm{C}$ genotypes between DM1 and non-diabetic subjects, DNA samples from patients were interrogated in parallel with the control DNA obtained from a large sample $(n=2133)$ of Czech non-diabetic population DNA bank established and maintained in IKEM.

To determine whether frequencies of $-765 \mathrm{G}>\mathrm{C}$ genotypes and GC alleles in patients with manifest nephropathy differ from Type 1 diabetic patients at low risk of this complication, we analyzed DNA samples and clinical parameters in patients with duration of diabetes $>10$ years. The diagnosis of manifest DN was based on detection of persistent proteinuria $>0.5 \mathrm{~g} / 24$ hours or albuminuria $>300 \mathrm{mg} / 24$ hours. The patients recruited later in the course of the disease with compromised renal function and/or need for renal replacement therapy were also considered as having manifest nephropathy provided that the history of kidney disease was consistent with clinical course of nephropathy and presence of diabetic retinopathy. The patients defined as subjects with low risk of nephropathy (LRDN) were required to be normoalbuminuric (urinary albumin excretion $<20 \mu \mathrm{g} /$ minute or $<30 \mathrm{mg} / 24$ hours), diabetes duration $>10$ years, maximal recorded blood pressure (BP) below 140/90, and no treatment with inhibitors of reninangiotensin system or antihypertensive agents. Retinopathy status was assessed based on indirect ophthalmoscopy or retinal photography. Informed consent was obtained from all subjects participating in the study; the study protocol followed the principles expressed in the Declaration of Helsinki and will be approved by local ethics committees.

Genomic DNA was prepared from frozen whole EDTA blood samples with the use of salting out method (Miller et al. 1988). Analysis of polymorphisms was performed using the polymerase chain reaction, restriction analysis, and electrophoresis on $10 \%$ acrylamide gel followed by staining with ethidium bromide and visualisation in UV light. Oligonucleotides 5'gct acc ttc agt gta cat agc and 5'ccg ctt get ttg tcc atc ag and restriction enzyme SsiI were used.

Routine physical measurements were performed by trained staff at the Diabetes Center IKEM. Laboratory parameters were determined in Central laboratories IKEM using routine laboratory methods.

The frequencies of $-765 \mathrm{G}>\mathrm{C}$ genotypes in general non-diabetic Czech population were significantly different $(\mathrm{P}=0.005)$ from the entire $\mathrm{DM} 1$ patient population. Type 1 diabetic patients demonstrated frequencies (CC 13 (1.7\%), GC 242 (31.1\%) GG 524 (67.3\%), whereas the healthy individuals (CC 88 (3.7\%), GC 647 (27.4\%) GG 1627 (68.9\%).

Out of 779 patients with DM1 screened and genotyped for the purpose of this study, 153 patients met the criteria for LRDN and 139 patients had manifest diabetic nephropathy. The clinical characteristics of the two patient groups are shown in Table 1. Compared with LRDN group, the patients with DN demonstrated male preponderance, were older and had longer duration of diabetes. As expected, they also demonstrated higher HBA1c, blood pressure, serum creatinine and triglyceride levels as well as more advanced retinopathy. There were no significant differences in total plasma cholesterol. Table 2 summarizes the frequencies of $-765 \mathrm{G}>\mathrm{C}$ genotypes and GC alleles. There were no differences in 
these polymorphisms between LRDN and DN patients. Moreover, the allele frequencies also did not differ between the groups.

Table 1. Clinical parameters in patients with low risk of nephropathy (LRDN) and patients with manifest diabetic nephropathy (DN).

\begin{tabular}{lcc}
\hline Parameter & LRDN & LRDN \\
\hline$n$ & 153 & 139 \\
Gender $(M / F)$ & $76 / 77$ & $95 / 44^{\dagger}$ \\
Age (years) & $35 \pm 13$ & $44 \pm 12^{\dagger}$ \\
Duration of diabetes (years) & $18 \pm 8$ & $25 \pm 9^{\dagger}$ \\
HBAlc (\%) & $6.6 \pm 0.1$ & $7.1 \pm 0.2^{*}$ \\
Hypertension (n, \%) & 0 & $137(99)$ \\
SBP (mm Hg) & $119 \pm 11$ & $141 \pm 24^{\dagger}$ \\
DBP (mm Hg) & $75 \pm 8$ & $83 \pm 13^{\dagger}$ \\
Treatment with RAAS (n, \%) & 0 & $105(76)$ \\
S-creatinine ( $\mu$ mol/l) & $82 \pm 1$ & $252 \pm 16^{\dagger}$ \\
Proliferative retinopathy $(n, \%)$ & $6(3.9)$ & $82(59)^{\dagger}$ \\
\hline
\end{tabular}

${ }^{*} p<0.05,{ }^{\dagger} p<0.01$ vs. LRDN.

Table 2. Frequencies of $-765 \mathrm{G}>\mathrm{C}$ genotypes and $\mathrm{GC}$ alleles in type 1 diabetic patients with low risk of nephropathy (LRDN) and manifest nephropathy (DN).

\begin{tabular}{lccccc}
\hline Group & \multicolumn{3}{c}{ Genotype } & \multicolumn{2}{c}{ Allele } \\
& GG & GC & CC & G & C \\
\hline$L R D N$ & $100(65)$ & $49(32)$ & $4(3)$ & $249(81)$ & $57(19)$ \\
$D N$ & $98(71)$ & $38(27)$ & $3(2)$ & $234(84)$ & $44(16)$ \\
\hline
\end{tabular}

Data are $\mathrm{N}(\%)$.

Unlike number of other polymorphisms in a variety of candidate genes, the $-765 \mathrm{G}>\mathrm{C}$ has an impact on COX-2 expression, on the generation of COX-2-derived prostanoids, and consequently on their effects in mediating inflammation (Papafili et al. 2002). Increased activity and/or expression of COX-2 has been described in the diabetic kidney both in clinical and experimental settings (Komers et al. 2001, Cherney et al. 2008). In addition, low grade inflammation has been implicated in the pathophysiology of this disorder (Utimura et al. 2003).
The study provided new information about distribution of $-765 \mathrm{G}>\mathrm{C}$ genotypes in Czech general population. Present data correspond to previous findings in Caucasian subjects in the UK (Papafili et al. 2002), with low frequency of CC homozygotes.

Importantly, our study did identify alterations in this distribution in patients with DM1 - it seems that rare $\mathrm{CC}$ homozygotes are protected against DM1 development. Considering the autoimmune etiology of ß-cell destruction in DM1, this finding should stimulate further studies exploring the roles of $\mathrm{COX}-2$ in the process.

Further analysis did not reveal any differences in these polymorphisms between the DM1 patients with manifest DN and patients at low risk of DN. This might be in part due to scarcity of CC genotypes in the population in general, requiring substantially higher number of participants. A previous study conducted in patients with myocardial infarction has indicated that carrying the $\mathrm{C}$ allele alone is associated with $\mathrm{CV}$ protection (Cipollone et al. 2004). However, the comparison of $\mathrm{C} / \mathrm{G}$ allele frequencies in our cohort of patients also did not reveal differences between the DN and LRDN groups.

One might argue that after $>10$ year cut-off for duration of diabetes the LRSD group of patients might still include patients at risk of developing nephropathy. Although this is possible, and nephropathy may develop after several decades of diabetes, patients with normoalbuminuria and normotension are already at a substantially lower risk during the second decade after the onset of the disease.

In summary, we found lower frequency of the protective $-765 \mathrm{G}>\mathrm{C}$ genotype in DM1 patients as compared to general population. However, the study did not identify any associations of this polymorphism with the development of nephropathy in DM1.

\section{Conflict of Interest}

There is no conflict of interest.

\section{Acknowledgements}

This work was funded by grant no. NR9368-3/2007 of the Internal Grant Agency, Ministry of Healthcare Czech Republic (RK, JH). The authors are grateful to T. Neskudla for technical assistance. 


\section{References}

CHERNEY DZ, MILLER JA, SCHOLEY JW, BRADLEY TJ, SLORACH C, CURTIS JR, DEKKER MG, NASRALLAH R, HEBERT RL, SOCHETT EB: The effect of cyclooxygenase-2 inhibition on renal hemodynamic function in humans with type 1 diabetes. Diabetes 57: 688-695, 2008.

CIPOLLONE F, TONIATO E, MARTINOTTI S, FAZIA M, IEZZI A, CUCCURULLO C, PINI B, URSI S, VITUlLO G, AVERNA M, ARCA M, MONTALI A, CAMPAGNA F, UCCHINO S, SPIGONARDO F, TADDEI S, VIRDiS A, CIABATTONI G, NOTARBARTOLO A, CUCCURULlO F, MEZZETTI A: A polymorphism in the cyclooxygenase 2 gene as an inherited protective factor against myocardial infarction and stroke. JAMA 291: 2221-2228, 2004.

FURUKADO S, SAKAGUCHI M, YAMAGAMI H, YAGITA Y, HOSHI T, ABE Y, HOUGAKU H, HORI M, SAKODA S, KITAGAWA K: Cyclo-oxygenase-2 -765G $>\mathrm{C}$ promoter variants are associated with lower carotid plaque echogenicity in Japanese. Cerebrovasc Dis 27: 91-98, 2009.

KOMERS R, ANDERSON A, EPSTEIN M: Renal and Cardiovascular Effects of Selective Cyclooxygenase-2 Inhibitors. Am J Kidney Dis 38: 1145-1157, 2001.

LINTON MF, FAZIO S: Cyclooxygenase-2 and atherosclerosis. Curr Opin Lipidol 13: 497-504, 2002.

MILLER SA, DYKES DD, POLESKY HF: A simple salting out procedure for extracting DNA from human nucleated cells. Nucleic Acids Res 16: 1215, 1988.

PAPAFILI A, HILL MR, BRULL DJ, MCANULTY RJ, MARSHALL RP, HUMPHRIES SE, LAURENT GJ: Common promoter variant in cyclooxygenase-2 represses gene expression: evidence of role in acute-phase inflammatory response. Arterioscler Thromb Vasc Biol 22: 1631-1636, 2002.

SMITH WL: The eicosanoids and their biochemical mechanisms of action. Biochem J 259: 315-324, 1989.

UTIMURA R, FUJIHARA CK, MATTAR AL, MALHEIROS DM, NORONHA IL, ZATZ R: Mycophenolate mofetil prevents the development of glomerular injury in experimental diabetes. Kidney Int 63: 209-216, 2003. 